\title{
ODÓN DE BUEN: CUARENTA Y CINCO AÑOS DE COMPROMISO CON LA UNIVERSIDAD
}

\author{
Alberto Gomis \\ Dpto. CC. Sanitarias y Médico-sociales. Universidad de Alcalá
}

\section{RESUMEN}

El naturalista aragonés Odón de Buen y del Cos fue durante veintidós cursos académicos catedrático de Historia Natural en la Universidad en Barcelona y durante veintitrés de la Universidad de Madrid. Firme defensor de la teoría evolucionista de Darwin, del trabajo experimental en el campo y en el laboratorio, en este artículo se ponen en valor sus esfuerzos, como docente, para popularizar las ciencias positivas y, así, separarlas de las preocupaciones, de la superstición y del fanatismo, que eran causas primordiales del atraso moral y material en que se encontraban en España.

PALABRAS CLAVE: Odón de Buen. Historia Natural. Universidad de Barcelona. Universidad de Madrid. Libros prohibidos.

\section{ODÓN DE BUEN: FORTY-FIVE YEARS OF COMMITMENT TO THE UNIVERSITY}

\begin{abstract}
The aragonese naturalist Odón de Buen y del Cos for twenty-two annual academic courses professor of natural history at the University of Barcelona and for twenty-three of the University of Madrid. Strong supporter of Darwin's evolutionary theory, experimental work in the field and laboratory, in this paper puts the value of their efforts, as an educator, to popularize the natural sciences and thus separated from the concerns, superstition and fanaticism, which they were basic reasons of the moral and material backwardness in which Spain was found.
\end{abstract}

KEY WORDS: Odón de Buen. Natural History. University of Barcelona. University of Madrid. Prohibited books.

\section{INTRODUCCIÓN}

El 5 de septiembre de 1889 Odón de Buen y del Cos tomaba posesión de la cátedra de Historia Natural en la Universidad de Barcelona. El propio Odón de Buen recordaría en sus Memorias el hecho de que al estar la mayor parte del 
personal de la Universidad disfrutando todavía de vacaciones, hubo de darle posesión de la misma un catedrático de Matemáticas de avanzada edad, que regentaba en esos momentos el decanato de la Facultad de Ciencias, hombre práctico y muy en armonía con el ambiente que entonces reinaba, que le dijo:

Entra Ud., compañero, en el escalafón muy joven y como tendrá Ud. la aspiración natural de llegar al primer número del escalafón, ahora a desempeñar puntual y correctamente la cátedra y a tomarse las menores molestias posibles para vivir mucho ${ }^{1}$.

Las ciencias naturales se encontraban en España, en esos momentos, en plena renovación. Una renovación que implicaba el abandono de los clásicos trabajos de clasificación sistemática y su sustitución por otros más dinámicos, en los que algunos autores, aquellos que aceptaban las tesis evolucionistas, planteaban el parentesco entre los seres vivos. Uno de los principales artífices de esta renovación va a ser Odón de Buen, de ahí que hasta el momento se hayan publicado, aunque no sean completamente definitivos, un buen número de trabajos sobre su vida y obra ${ }^{2}$ y que se haya abordado, en detalle, su actuación en diversas parcelas, fundamentalmente como fundador del Instituto Español del Oceanografía ${ }^{3}$ y como uno de los más firmes defensores del darwinismo en España, lo que le valió la excomunión por parte del obispo de Barcelona, el cardenal Salvador Casañas ${ }^{4}$, pero también su participación co-

1 Buen, O. de. (2003), Mis memorias (Zuera, 1863 - Toulouse, 1939), transcritas del manuscrito original por María del Carmen de Buen López de Heredia, Zaragoza, Institución Fernando El Católico, p. 52.

2 Bolívar y Pieltain, C. (1945), Odón de Buen 1863-1945, Ciencia (México), 6, pp. 310-312; Bosque Maurel, J. (2003-2004), Odón de Buen, Boletín de la Real Sociedad Geográfica, 139-140, pp. 267-292; BuJOSA I HOMAR, F. y GLICK, Th.F. (1995), Odón de Buen y del Cos. L'oceanografia. En CAmarasa, J.M. y RocA, A. (dirs.), Ciència i Tècnica als Països Catalans: una aproximació biogràfica. Als darrers 150 anys, Barcelona, Fundació Catalana per a la Recerca, 1, pp. 761-791; CANO, J.L. (2003), Odón de Buen el republicano de los mares, Zaragoza, Xordica; GLICK, Th.F. (1991), La ciencia contemporánea en las Memorias de Odón de Buen. En Valera, M. y López FernándeZ, C. (eds.), Actas del V Congreso de la Sociedad Española de Historia de las Ciencias y de la Técnica, Murcia, SEHCT, vol. I, pp. 229-243; PAllás, R. [s.f.], Biografía. En BuEN, O. de, El concepto de la naturaleza, Barcelona, Salvador Manero Bayarri editor, pp. 5-26.

3 PÉRez de Rubin, J. (2004), $90^{\circ}$ Aniversario. El Instituto Español de Oceanografía. Sus orígenes y primeras investigaciones, Madrid, IEO.

4 ARQUÉs, J. (1984), Els veritables fets sobre la suspensió del cientific darwinsita Odón de Buen de la seva càtedra de la Universitat de Barcelona. En Hormigón, M. (ed.), Actas II 
mo naturalista en el viaje de la fragata $B l a n c a^{5}$, sus expediciones en pos del reconocimiento natural del África hispana ${ }^{6}$, la correspondencia que mantuvo con el Archiduque Luis Salvador ${ }^{7}$, las obras de Historia Natural que publicó en el siglo XIX ${ }^{8}$, e incluso, su actuación como uno de los naturalistas españoles que tuvieron que exiliarse a México tras la guerra civil ${ }^{9}$.

Por lo que respecta a la enseñanza de las ciencias naturales, o más bien de la historia natural como entonces se llamaba, hay que señalar que había sido fundamentalmente memorística, descriptiva y sistemática a lo largo del siglo XIX. En los libros de textos abundaban las prolijas clasificaciones y los nombres complejos, siendo frecuente que entre ellos se intercalaran pasajes bíbli$\cos$. Hay que tener en cuenta que en ese año, de 1889, sólo se podían completar los estudios universitarios de la Sección de Naturales en la Facultad de Ciencias de la Universidad de Madrid y esto explica el escaso número de

Congreso de la Sociedad Española de Historia de las Ciencias, Zaragoza, SEHC, vol. 1, pp. 285-303; ARQuÉs, J. (1985), Darwinisme i antidarwinisme a la Universitat de Barcelona: la suspensió d'Odón de Buen de la seva càtedra. En ARQuÉs, J., Cinc estudis sobre la Universitat de Barcelona (1875-1895), Barcelona, Columna, pp. 19-66; Gomis, A. y JosA, J. (2010), Odón de Buen y Charles Darwin, Zuera, 1, pp. 20-21.

5 Puig-SAmper, M.A., Fernández Pérez, J. y Marrogán Serrano, M.D. (1984), El viaje de la fragata Blanca (1886). En Hormigón, M. (ed.), Actas II Congreso de la Sociedad Española de Historia de las Ciencias, Zaragoza, SEHC, vol. 3, pp. 287-296.

6 GonzÁlez Bueno, A. y Gomis Blanco, A. (2002), Los naturalistas españoles en el África hispana (1860-1936), Madrid, Organismo Autónomo Parques Nacionales (Ministerio de Medio Ambiente); González Bueno, A. y Gomis Blanco, A. (2007), Los territorios olvidados. Estudio histórico y diccionario de los naturalistas españoles en el África hispana (1860-1936), Aranjuez, Doce Calles.

7 Bujosa I HOMAR, F. (1994), La correspondència entre Odón de Buen y del Cos i 1'Arxiduc Lluís Salvador, Bolleti de la Societat Arqueològica Lul-liana, 50, pp. 527-548.

8 Gomis, A., Josa, J., Fernández, J. y Pelayo, F. (1988), Historia Natural. Catálogo ilustrado siglos XVIII y XIX, Madrid, MEC.

9 Gomis Blanco, A. (2001), Los naturalistas españoles exiliados en México. En SÁNCHEz DíAz, G. y GARCía DE LEÓn, P. (coords.), Los científicos del exilio español en México, Morelia, Universidad Michoacana de San Nicolás de Hidalgo, pp. 167-200; Gomis BlANCO, A. (2008), Los socios de la Española exiliados en México. En PEREjón Rincón, A. y OuteRelo Domínguez, R. (eds.), Actas XVII Bienal de la Real Sociedad Española de Historia Natural. Encuentro entre los naturalistas a las orillas del Atlántico: Interrelaciones e influencias (México-España), Madrid, RSEHN, ICML y SMHN, pp. 185-193; SÁNCHEZ CARRILlO, S. (2001), Los oceanógrafos españoles en el exilio: la familia De Buen y sus aportaciones a la ciencia española y mexicana. En SÁnCheZ ANDrÉS, A. y Figueroa ZAMUdio, S., De Madrid a México. El exilio español y su impacto sobre el pensamiento, la ciencia y el sistema educativo mexicano, México, UMSNH y CAM, pp. 161-208. 
catedráticos de Naturales que figuraban en el escalafón cuando Odón de Buen ingresó en el mismo ${ }^{10}$.

En este trabajo se trata de probar como Odón de Buen, que contaba en esos momentos 25 años, hizo caso omiso al consejo del catedrático de Matemáticas, emprendiendo a partir de ese momento una formidable tarea en pos de la transformación de la enseñanza de las ciencias naturales, una transformación que ya anunció al impartir su primera clase, donde trazó el plan que quería seguir en el curso y que, según expuso, comprendía lecciones orales, prácticas en el laboratorio, nada más que fuera posible, y excursiones al campo para estudiar la naturaleza en la naturaleza misma ${ }^{11}$. Una nueva enseñanza de las ciencias naturales, bajo perspectiva biológica y evolucionista, actuación que ha sido escasamente tratada ${ }^{12}$.

Hasta esos momentos, la actividad científica más relevante de Odón de Buen había sido su participación, como naturalista, en el viaje de circunnavegación, con objeto de la instrucción de guardiamarinas, emprendido por la fragata Blanca en 1885, intervención que resultaría decisiva para su inclinación futura hacia la oceanografía. El viaje fue origen de su libro De Kristianía á Tuggurt: (impresiones de viaje) publicado en $1887^{13}$, el mismo año que alcanzó el doctorado. En el segundo semestre del año siguiente, 1888, publica el que debemos considerar su primer texto docente, una Cartilla de Historia Natural ${ }^{14}$ que dedica «A los Maestros» y que cree «útil para infiltrar en la niñez los sanos principios en que hoy se funda el estudio de la naturaleza, y para desarrollar el espíritu de observación, sin perjuicios y sin errores fundamentales» ${ }^{15}$.

10 Entre los últimos en incorporarse estaban: Ignacio Bolívar (1877), Alberto María de Segovia y Corrales (1881), Francisco Vidal y Careta (1885) y Emiliano Rodríguez y Risueño (1889).

11 BUEN (2003), pp. 52-53.

12 Fernández Malanda, D. (1998), La enseñanza de las ciencias naturales y Odón de Buen. En UnIVERSIDAD DE MuRCiA, La Universidad en el siglo XX: España e Iberoamérica: $X$ Coloquio de Historia de la Educación, Murcia, Universidad de Murcia, pp. 358-364.

13 Buen, O. de. (1887), De Kristianía á Tuggurt: (impresiones de viaje), Madrid, Imprenta de Fortanet.

14 Buen, O. de. (1888), Cartilla de Historia Natural, Madrid, José Matarredona [El Porvenir Editorial].

$15 \operatorname{BuEN}(1888)$, p. V. 


\section{EL PRIMER INTENTO DE SER CATEDRÁTICO DE UNIVERSIDAD}

Unos meses antes de ganar la cátedra de Barcelona, Odón de Buen había opositado a la de Historia Natural de la Facultad de Ciencias de la Universidad de Valladolid. Sin embargo, en este primer intento de acceder a una cátedra no tuvo éxito, ya que, tras unas reñidísimas deliberaciones, el tribunal se decantó a favor de Emiliano Rodríguez Risueño, otorgando a este cuatro votos, frente a los tres que obtuvo Odón de Buen ${ }^{16}$.

La cátedra vallisoletana se había convocado por R. O. de 6 de agosto de 1887 (Gaceta de Madrid, 12-VIII-1887). Entre los miembros del tribunal para juzgarla, que inicialmente designó el Consejo de Instrucción Pública, hubo un fallecimiento y dos renuncias ${ }^{17}$, actuando finalmente Manuel $\mathrm{M}^{\mathrm{a}}$ Jose de Galdo, como presidente, y los vocales Antonio Orio, José Arevalo y Baca, Serafín Saéz y Aguado, Alberto Segovia Corrales, José Ma Solano y Eulate y Santiago Bonilla. En el plazo reglamentario, firmaron la instancia para concurrir a los ejercicios que debían proveer la cátedra siete opositores, Odón de Buen, Emiliano Rodríguez Risueño, Félix Gila y Fidalgo, Apolinar Federico Gredi1la, Ventura Reyes Prósper, José Bonet y Andreu y Aniceto Llorente y Arregui, si bien - en última instancia- sólo se presentaron los cuatro citados en primer lugar.

Como era preceptivo, por tratarse de una cátedra experimental, los cuatro opositores practicaron los cuatro ejercicios que el Reglamento señalaba para éstas y que se describen con más detalle en el apartado siguiente. Dichos ejercicios se llevaron a cabo en el madrileño Instituto del Cardenal Cisneros desde el día 22 de marzo de 1888, en que se sortearon las dos bincas (una quedó integrada por Gila y Rodríguez; la otra por Buen y Gredilla), hasta el 26 de abril, en que la segunda binca finalizó el cuarto ejercicio. Tres días más tarde se reunió el Tribunal para proceder a la votación que debía determinar el opositor que había de ser propuesto para la cátedra vacante, con el resultado ya expresado. A continuación, en sucesivos votaciones, se procedió a determinar el mérito relativo de los demás opositores, resultando:

16 Expediente para proveer por oposición la cátedra de Historia Natural vacante en 21 de junio de 1887 por posesión de D. Augusto González de Linares de la Dirección de Estación de biología Marítima. Archivo General de la Administración (AGA), signatura (05) 32/07435.

17 El fallecimiento de José Planellas Giralt y las renuncias de Vicente González Canales y de Salvador Calderón y Arana. Este último, inicialmente, figuraba como primer suplente. 
$\mathrm{N}^{\mathrm{o}} 1$ Don Odón de Buen, por seis votos, contra uno que alcanzó Don Apolinar Federico Gredilla.

$\mathrm{N}^{\circ} 2$ Don Apolinar Federico Gredilla y Gauna, por unanimidad.

$\mathrm{N}^{\mathrm{o}} 3$ Don Félix Gila y Hidalgo por cuatro votos, contra tres papeletas en blanco ${ }^{18}$.

Trascurrido casi siglo y cuarto de su celebración y conociendo, como hoy conocemos, lo que cada uno de los cuatro opositores fue capaz de hacer en el terreno científico a lo largo de su vida, no deja de sorprendernos el resultado de aquella oposición. Odón de Buen lo explicaba del modo siguiente:

[...] las oposiciones fueron regocijantes y alguna vez tumultuosas, iba a ellas mucho público de estudiantes, me tocó en línea, por suerte, con el candidato de la mayoría del tribunal. Me ensañé con él, con el programa que había presentado, con sus explicaciones confusas y torpes de palabra y enrevesadas de conceptos, con la incultura que demostró en los problemas fundamentales... Tal fue la actuación de mi contrincante que uno de los jueces, que iba dispuesto a votarle, se retiró antes de la votación, confesando que no podía hacerlo, y así en el juicio resultaron tres votos a mi favor y tres en contra. Se repitió la votación con el mismo resultado. Me consultó el presidente, que me había votado, si hacía valer su voto de calidad; yo le agradecí sus buenas intenciones pero rogándole influyese, como así lo hizo, para que fuesen pronto las oposiciones a la cátedra de Barcelona que era la que yo ambicionaba. En una votación final lograron los tres jueces contarios a mí que tomara parte el cuarto que se había retirado y la bomba final fue que el pretencioso quedó sin cátedra y le dieron por cuatro votos a un modesto condiscípulo que había estado al margen discretamente y que era pariente o muy amigo del juez arrepentido ${ }^{19}$.

Sea como fuere, Emiliano Rodríguez Risueño pudo tomar posesión de la cátedra de Historia Natural en la Universidad de Valladolid, mientras que Odón de Buen y Félix Gila, junto con algún otro, como ahora veremos, comenzaron a preparar la convocada en Barcelona, precisamente durante la celebración de ésta.

\section{LA OBTENCIÓN DE LA CÁTEDRA DE BARCELONA}

En efecto, con fecha del 28 de marzo de 1888 la Gaceta de Madrid anunciaba la vacante de la cátedra de Historia Natural en la Facultad de Ciencias, Sección de Naturales, de la Universidad de Barcelona. Dotada con el sueldo

18 Cfr. Expediente citado en la nota 16.

19 BUEN (2003), pp. 51-52. 
anual de 3.500 pesetas, debía proveerse por oposición, con arreglo a lo que prevenía el artículo 226 de la Ley de Instrucción pública vigente, la célebre Ley Moyano de $1857^{20}$. La cátedra había quedado vacante tras el fallecimiento de José Planellas Giralt, acaecido el 1 de febrero de ese año.

El tribunal que debía juzgarla se reunió, por vez primera, el 26 de junio del año siguiente, 1889, en el madrileño Museo de Historia Natural. Presidido por Ignacio Bolívar, formaban parte del mismo Joaquín González Hidalgo, Eduardo Lozano, Lucas Mallada, Salvador Calderón y Carlos Mazarredo como vocales, siendo Francisco Quiroga el Secretario del mismo. El 2 de julio citaron a los firmantes de la oposición en el Salón de Grados de la Facultad de Ciencias de la Universidad Central, donde fueron llamados, por el Secretario, en el orden siguiente: Aniceto Llorente y Aregui (no compareció por enfermedad, pero estuvo representado); Francisco de Sales de Delás y de Gayola; Ventura Reyes Prósper; Félix Gila y Fidalgo; José Gogorza y González; José Bonet y Andreu (no compareció); Telesforo Aranzadi y Unamuno; y Odón de Buen y del Cos. No nombró a Blas Lázaro Ibiza, por haber renunciado a la misma. En aquella sesión se procedió al sorteo de las discusiones, resultando: trinca: Gogorza, Reyes y Delás; binca 1: Aranzadi y Gila; binca 2: Buen y Llorente ${ }^{21}$.

El día 5 los miembros del Tribunal procedieron a la lectura y discusión de las 120 preguntas que habían quedado en redactar entre todos ellos, correspondiendo la mitad de ellas a zoología, y la otra mitad a mineralogía, botánica y geología. A partir del día siguiente, 6 de julio, comenzó el primer ejercicio, que consistía en la extracción al azar ${ }^{22}$, por cada uno de los opositores, de diez preguntas de las seleccionadas por el Tribunal, a las que debían contestar seguidamente. El tiempo que emplearon, cada uno de ellos, en responder a las que les habían correspondido, osciló entre la hora y siete minutos de Aranzadi y la hora y veintiún minutos de Gogorza. Las renuncias de Gila y de Llorente, comunicadas por el Presidente el día 8, modificó la composición de la trinca y las bincas. Odón de Buen pasó entonces a emparejarse con Telesforo Aranzadi.

20 Ley de Instrucción pública de 9 de septiembre de 1857 (Gaceta de Madrid, 10-IX1857). El artículo 226 señalaba: «De cada tres plazas vacantes de Catedráticos numerarios se proveerán dos en supernumerarios, mediante concurso y á propuesta del Real Consejo de Instrucción pública, y una por oposición».

21 Expediente para proveer por oposición la cátedra de Historia Natural vacante en $1^{\circ}$ de marzo de 1888 por defunción de Don José Planellas y Giralt. AGA, Educación y Ciencia (05) $32 / 07434$.

22 De una bolsa que contenía tantas bolas numeradas como preguntas había. 
El día 9 comenzó el segundo ejercicio, consistente en la extracción por cada opositor, al azar, de tres temas de los que cada uno de ellos había presentado en su programa. Se le incomunicaba toda la noche, junto a una serie de libros que solicitaba para prepararlo, y al día siguiente lo defendía en la binca o la trinca correspondiente. El día que llegó el turno a Odón de Buen, el 14 de julio, eligió la lección 113 de su programa, que llevaba por enunciado «Acción geológica de los seres vivos. Efectos protectores y destructores. Formación de las rocas. Formaciones madrepóricas. Islas y arrecifes de coral. Su formación».

El tercer ejercicio tenía como objeto la defensa del programa por cada opositor, frente al rival, o los rivales que le hubieran caído en suerte. Y el cuarto, el ejercicio práctico, a su vez estaba dividido en dos partes: una primera, con libros, consistente en la clasificación por cada opositor de los diez objetos que les fueran sorteados, tratando de llegar a la determinación de especie, en un tiempo máximo de cuatro horas; la segunda, sin libros, examen de veinte objetos en un tiempo máximo de una hora.

El 22 de julio se reunió el Tribunal, de nuevo en el Museo, para verificar la votación para la propuesta, resultando ésta favorable a Odón de Buen por unanimidad de seis votos, pues el vocal Joaquín González Hidalgo se había retirado del mismo doce días antes. La calificación de los demás opositores, por mérito, situó en primer lugar a Gogorza, en segundo a Reyes Prósper, tercero a Delás y cuarto a Aranzadi.

\section{LOS PRIMEROS TEXTOS UNIVERSITARIOS}

Como empezábamos señalando, una vez que Odón de Buen tomó posesión de la cátedra, emprendió una formidable tarea en pos de la transformación de la enseñanza de las ciencias naturales. La realización de excursiones pudo comenzarlas casi de inmediato, pero pasó bastante tiempo hasta que consiguió organizar ejercicios prácticos. Por lo que respecta al libro de texto, aunque no era partidario de imponer como texto libro alguno, comenzó la confección de su «Historia Natural» para universidades. Consideraba que el libro era «el mejor auxiliar y la guía mejor del estudiante si refleja las tendencias de la cátedra y contiene los indispensables conocimientos para cumplir el programa del curso; puede, ampliando los temas suficientemente, servir de obra de consulta aún después de terminada la carrera» ${ }^{23}$.

23 BUEN (2003), p. 59. 
El objeto que se propuso al iniciar la publicación de un «Curso completo de Historia Natural» era ofrecer una síntesis completa de los progresos y estado actual de esta ciencia. Lo haría en tres volúmenes, el primero dedicado a la Geología, el segundo - el más amplio - a la Zoología, y el tercero a la Botánica. En 1890, mediante cuadernos comenzó a publicar, en el establecimiento tipográfico «La Academia» su Tratado elemental de Geología, que conformó un volumen de cuatrocientas páginas, con diez láminas litografiadas sin paginar y numerosos grabados en el texto ${ }^{24}$. La obra, dedicada «al eminente geólogo D. José Macpherson», surge en un momento en que no existía libro de autor español dedicado a la enseñanza universitaria de la disciplina. Era deseo de Odón de Buen: «hacer una Geología española sintetizando lo que en nuestro país, y acerca de nuestro país, se ha escrito» ${ }^{25}$. Por ello, según señala el propio autor:

Tiene la obra marcado carácter didáctico; he sacrificado mucho á la claridad. Además es elemental por su extensión; supera, sin embargo, á la necesaria para la enseñanza á que está dedicada, y me ha sido forzoso este superavit, que es preferible a un déficit, y por otra parte hace útil la obra à muchas otras personas que no son estudiantes. También he procurado hacerle comprensible á toda inteligencia mediana; hacen falta en nuestro pueblo muchos libros que popularicen las ciencias positivas para arrancarle las preocupaciones, la superstición y el fanatismo, causas primordiales de su atraso moral y material ${ }^{26}$.

El Tratado está dividido en cuatro partes, antes de las cuales figuran unos Preliminares, donde aclara qué es la geología, cuál es su objeto y hace una breve reseña histórica, donde apunta como: «El largo período de la dominación teocrática mantuvo á todos los conocimientos sujetos al yugo del escolasticismo y á los moldes primitivos de una cosmogonía, forjada por pueblos semi-salvajes é impuesta á título de divina á los sabios de muchas generaciones ${ }^{27}$. Apunta, a continuación, los nombres más ilustres que han contribuido a formar «una ciencia de tantos vuelos como la Geología». Desde Smith, en Inglaterra; Haüy, Romé de l'Isle, Cuvier y Brongniart, en Francia; Humboldt y de Buch, en Alemania hasta «las sencillas teorías de Malett, Suess, Neumayer y Macpherson, que vienen á colocar la vida de la tierra dentro de los prin-

24 BuEn, O. de (1890), Tratado elemental de Geología, Barcelona, Tipografía La Academia de Viuda e Hijos de E. Ullastres y $\mathrm{C}^{\mathrm{a}}$.

25 BUEN (1890), p. XV.

26 BUEN (1890), p. XVI.

27 BUEN (1890), pp. 7-8. 
cipios transformistas del inmortal Darwin» ${ }^{28}$. Terminan estos preliminares con un apartado sobre la geología en España. La primera parte de la Geología se dedica a la morfología terrestre, la segunda a la litología, la tercera a la dinámica terrestre y, la cuarta y última, a la geología histórica.

El Tratado, del que deberemos ocuparnos más adelante por ser incluido en la lista de obras prohibidas, tuvo una buena acogida y mereció juicios halagüeños y el favor por parte de algunos profesores de universidades americanas de lengua española que la declararon de texto ${ }^{29}$. La segunda edición de esta Geología, ahora en la Imprenta Gutenberg, vería la luz en 1896.

Conforme al plan previsto, siguieron a este Tratado elemental de Geología, el Tratado elemental de Zoología y el Tratado elemental de Botánica. El primero salió de los talleres de «La Academia» también en ese año de 1890, mientras que el Tratado elemental de Botánica no vería la primera edición hasta 1896. Fue por recomendación del político e intelectual republicano Francisco Pi y Margall (1824-1901) que Odón de Buen entrara en relación con este establecimiento tipográfico. Al frente de dicho establecimiento figuraba Fargas Pellicer, de justa fama en la vida obrera internacional, y ejercía de corrector de pruebas Anselmo Lorenzo, apóstol en España de las ideas de Bakunin. Allí conoció a Manuel Soler, quien al poco tiempo pasaría a ser su editor ${ }^{30}$.

\section{LAS EXCURSIONES DESDE BARCELONA}

Desde el primer curso académico, con grupos de veinte, o a lo sumo cuarenta alumnos, comenzó las excursiones por los alrededores de Barcelona, llegando de un lado hasta Gavá y Castelldefels, del otro hasta Papiol, recorriendo la pintoresca mole del Montserrat. Excursiones en las que el principal objetivo era que los alumnos vieran los seres vivos en su propio medio ${ }^{31}$. Oigamos al maestro:

Estudiar la Naturaleza en la Naturaleza misma ha sido siempre mi afán. Conocer los seres naturales en las colecciones, formadas con arreglo a una clasificación cualquiera, disecados con mayor o menor fidelidad, no es conocer la Naturaleza;

\footnotetext{
28 BUEN (1890), p. 9.

29 BUEN (2003), p. 59.

30 BUEN (2003), p. 59.

31 BUEN (2003), p. 55.
} 
en ésta viven, se agitan, se asocian, con letras de un alfabeto que unidas forman palabras y las palabras oraciones y las oraciones representan pensamientos y los pensamientos reflejan leyes naturales que interpretan el complejo fenómeno de la vida, con sus modalidades, con sus asociaciones, con sus adaptaciones necesarias ${ }^{32}$.

Al poco tiempo, con el fin de ampliar el campo de sus excursiones, entró en contacto con el reputado zoólogo francés Henri de Lacaze-Duthiers (18211901), fundador y director del Laboratorio Aragó, establecido en la localidad francesa de Banyuls-sur-Mer, a pocos kilómetros de la frontera española. En el curso 1894-95 formó parte, con un grupo de alumnos de Zoología, de una excursión que profesores y alumnos franceses hicieron a Banyuls y alrededores y, a partir de entonces, las visitas al centro con sus alumnos se repetían cada año y, por lo general, en más de una ocasión. Visitas al Laboratorio Aragó en las que los alumnos hacían prácticas con animales marinos, empleando los poderosos medios de que disponía el establecimiento ${ }^{33}$.

Pero como carecía de todo tipo de subvención, hubo de dividir las excursiones en dos clases: obligatorias y voluntarias. Las primeras, que no conllevaban ningún gasto, se verificaban algunos días de fiesta por los alrededores de Barcelona y en secciones de 25 alumnos. Las excursiones voluntarias las llevaba a cabo, tan sólo, con aquellos alumnos que satisfacían los exiguos gastos. Antes de partir, en la cátedra, daba una sesión teórica y otra sesión práctica de lo que deberían ver durante la excursión, de este modo también se instruían algo los alumnos que no podían acudir. Cada curso académico, Odón de Buen editaba el programa de la asignatura en el que, además de incorporar las disposiciones vigentes, incluía el plan del curso, detallándose las excursiones que, de uno y otro tipo, se iban a efectuar. Así, por ejemplo, en el curso 1901-02 se programaron las siguientes excursiones voluntarias:

En Noviembre, durante los días 1, 2 y 3, al «Laboratorio Aragó» en Banyulssur-Mer.

En Diciembre, a la desembocadura del Ebro.

En Semana Santa y Pascua, á la isla de Mallorca.

En algunos días festivos de primavera, se organizarán excursiones marítimas para dragar por la costa de la provincia de Gerona, aprovechando el arte de arrastre denominado $b_{o u}{ }^{34}$.

32 BUEN (2003), p. 55.

33 Buen, O. de (1899), Programa de un curso de zoología. $8^{a}$ edición. Curso de 1899 á 1900, Barcelona, Manuel Soler, p. 9.

34 BuEn, O. de (1901), Programa del curso de zoología. Curso de 1901 á 1902, Barcelo- 
El viaje que todos los años realizaba a la isla de Mallorca era de gran interés, por la riqueza de su fauna. En «aquel hospitalario y hermoso país», como califica Odón de Buen a sus gentes y a sus tierras, contaba con los medios y ayudas para la recolección de animales pertenecientes a la mayor parte de los tipos zoológicos. Señalaba el catedrático de la Universidad de Barcelona cómo: «En nuestras excursiones á la gruta de Artá, á las salinas de L'Avall, á Miramar. etc., hacemos recolección abundante de animales terrestres, que me permiten dar lecciones sobre el terreno» ${ }^{35}$. En muchas ocasiones, el viaje a la isla de Mallorca tenía como complemento la visita al archiduque Luis Salvador (1847-1915), en la bella finca que éste tenía en Miramar ${ }^{36}$.

También, en ocasiones, a la vuelta de la excursión se verificaba una sesión oficial y pública en la que algunos de los alumnos participantes y el propio profesor, daban cuenta de la misma. Así sucedió en la que se celebró el domingo 17 de marzo de 1895, en la sala doctoral de la Universidad de Barcelona bajo la presidencia del decano de la Facultad de Ciencias, José Ramón de Luanco, en la que uno de los escolares leyó el «Diario de Viaje», redactado por ellos mismos; otro, las notas tomadas por los excursionistas de medicina acerca de los hospitales y demás establecimientos benéficos; mientras que el propio de Buen explicó las observaciones, estudios, experimentos y demás resultados prácticos que habían logrado sus discípulos en la citada excursión ${ }^{37}$.

En alguna oportunidad se dirigieron a las más altas cumbres del Montseny. El itinerario seguido transcurría por Breda, Santa Fe, San Marsal, Matagalls, Viladrau, Argucias y Hostalrich. El objetivo era, en esta ocasión, que los excursionistas estudiaran los animales que vivían en las grandes altitudes. No era una excursión sencilla, pero sí resultaba muy económica ${ }^{38}$.

Pero el viaje que el propio Odón consideró como el de mayor envergadura, fue el que, a mediados de la década de los noventa, llevó a cabo a Italia con medio centenar de alumnos. El viaje de ida y vuelta lo realizaron en vapores de la línea de Buenos Aires a Génova, que hacía escala en Barcelona. Visitaron Génova, Milán, Pisa, Roma y Nápoles. Tenían como objetivo principal estudiar la región volcánica del Vesubio, pero aprovecharon el viaje con visitas a los Museos de Roma y Nápoles, así como en el Laboratorio de Biología

\footnotetext{
na, Universidad de Barcelona, Facultad de Ciencias, Manuel Soler, p. 9.

35 BUEN (1899), p. 9.

36 BUEN (2003), p. 56.

37 La Vanguardia, 16 de marzo de 1895, p. 2.

38 BuEN (1899), p. 9.
} 
Marina de esta última ciudad. Odón de Buen tenía proyectado llegar hasta Ginebra y el glaciar de Chamonix, de ahí el título que había dado a la excursión «Del fuego y el hielo», pero no contaron con suficiente tiempo, entre vapor y vapor, para acercarse a los glaciares ${ }^{39}$.

\section{LAS PRÁCTICAS EN LA UNIVERSIDAD DE BARCELONA}

La carencia de medios y de recursos económicos para la realización de clases prácticas con que se encontró Odón de Buen al acceder a la cátedra se prolongó en el tiempo. Durante los primeros cursos, tan sólo pudo hacer la exposición de algunos ejemplares durante la explicación teórica. De ahí, el lamento que figura en el programa de Zoología del curso 1899 a 1900:

Ni dispongo por ahora en la Universidad de material suficiente, ni de las condiciones apropiadas para las lecciones prácticas; no permite hacerlas tampoco, en la medida que la Ciencia exige, el gran número de alumnos matriculados en esta asignatura.

Por estas razones, invito á los estudiantes á que hagan las prácticas en casa, presentándome las preparaciones anatómicas para corregirlas. Pueden hacerlo si siguen las indicaciones de mi Tratado elemental de Zoología ( $3^{\text {a }}$ edición), fruto de larga práctica personal. Para la disección, les bastan: un bisturí, una tijera y unas pinzas; para la observación microscópica, es necesario un microscopio que aumente por lo menos 500 diámetros $^{40}$.

Sin embargo, esta precaria situación iba a cambiar dos cursos más tarde, cuando vencidas en su mayor parte las dificultades de organización y ampliado el tiempo disponible gracias a la luz eléctrica, se organizaron las prácticas de los alumnos matriculados en secciones de cincuenta alumnos. A ello contribuyó el R. D. de 4 de agosto de 1900 (Gaceta de Madrid, 7-VIII-1900) que disponía que, en las asignaturas cuyo pago requiriese instrumental que pudiese sufrir deterioro y ocasionasen gastos, los alumnos abonarían, al tiempo de matricularse, una cuota igual a la mitad de los derechos de la matrícula de cada asignatura. Los derechos se fijaron en 10 pesetas por asignatura y se introdujeron, experimentalmente, en el curso 1900-01 en algunas asignaturas de la Facultad de Ciencias ${ }^{41}$. Los martes, jueves y sábado, en el Laboratorio y

\footnotetext{
39 BUEN (1899), p. 10. y BUEN (2003), pp. 56 y 57.

40 BUEN (1899), p. 8.

41 BARATAS, A. (1997), Introducción y desarrollo de la biología experimental en España entre 1868 y 1936, Madrid, CSIC, p. 122.
} 
a la hora señalada, iban sucediéndose las secciones por orden riguroso. Cada sección se subdividía en grupos de diez alumnos, figurando al frente, de cada uno de ellos, un jefe de trabajo, designado por la Facultad en concurso abierto entre los doctores, licenciados y antiguos alumnos de Zoología que hubieran obtenido la calificación de sobresaliente en la asignatura. Cada alumno estaba provisto de una libreta de prácticas donde anotaba los ejercicios que realizaba y donde se reflejaba la nota que su trabajo merecía. La no asistencia a tres prácticas consecutivas, si no eran compensadas por tres días de excursión al campo, les inhabilitaba para ser admitidos a los exámenes ordinarios ${ }^{42}$.

El cuestionario de ejercicios prácticos, del programa del curso 1901 a 1902, comprendía 26 ejercicios, que iban desde la preparación de células epiteliales vibrátiles y pavimentosas, hasta la determinación de invertebrados de la fauna catalana y vertebrados de nuestra fauna, pasando por el aislamiento del aparato reproductor y del sistema nervios del caracol, o la preparación de un esqueleto de rana, o parte de él, entre otras ${ }^{43}$.

LA PROHIBICIÓN DE SU TRATADO ELEMENTAL DE GEOLOGÍA Y DE SU TRATADO ELEMENTAL DE ZOOLOGÍA

El Obispado de Barcelona vio con preocupación lo que se decía en algunos párrafos de dos obras de Odón de Buen, concretamente de su Tratado elemental de Geología y de su Tratado elemental de Zoología, consiguiendo que ambas fueron condenadas por el Papa León XIII por decreto pontificio de 15 de junio de 1895. Las dos ediciones sancionadas eran las publicadas por la Editorial «La Academia» en 1890 y ambas, según el decreto pontificio, tamquam praedammatum in Regulis Indicis ${ }^{44}$. Al ser incluidas, ambas obras, en el Índice de libros prohibidos, no era lícito a ningún católico leer ni retener dichas obras.

La prensa nacional se hizo eco de la prohibición, alineándose unas publicaciones a favor de la libertad de cátedra, y por tanto de Odón de Buen, y otros en contra. Así, con la firma de Teófilo Nitrám se publicó en La lectura dominical el artículo «¡Cosas de España!» en donde se destacaba la «plancha» tan estrepitosa que acababan de «tirarse» los miembros del Consejo de Instrucción Pública y el Ministro de Fomento al enviar un oficio aprobando

42 BUEN (1901), pp. 8-9.

43 Buen (1901), pp. 28-31.

44 Decretum. Feria VI, die 14 Junii 1895. 
las obras Tratado elemental de Geología y Tratado elemental de Zoología, que habían sido condenadas por León XIII ${ }^{45}$.

A la semana siguiente, el semanario vuelve a la carga y, entonces, en la sección «Polémica Religiosa», que se publica sin firma, advierten:

Confiamos en que no prevalecerán esos textos malditos, que ningún católico puede leer, y que el catedrático que, en una nación católica, ataca desde la cátedra la Religión del Estado, será separado como reo de lesa inteligencia. No se toleraría un catedrático que enseñase el anarquismo ó predicase el derecho al robo, y no debe permitirse un Odón de Buen que, enseñando el ateismo, arroja en cientos de jóvenes la semilla de toda maldad. La cátedra debe ser cátedra de instrucción, no de destrucción é inmoralidad ${ }^{46}$.

A los pocos meses de iniciarse el curso 1895-1896, una asociación de pretendidos padres de familia de Barcelona, la Associació de Pares de Familia ${ }^{47}$, ideó un plan contra Odón de Buen. Resucitaron, entonces, una vieja disposición de la Ley Moyano, aquella que impide impartir enseñanzas contrarias a los dogmas de la religión católica, y pidieron al Rector, el farmacéutico Julián Casaña (1833-1911), que fuera despedido de sus funciones por faltar a ella. Una disposición timorata, de éste, fechada el día 6 de octubre, suspendía provisionalmente al catedrático de la enseñanza.

A la mañana del día siguiente, al dirigirse Odón a dar clase, le esperaba en la plaza de la Universidad una gran muchedumbre de gente, que le acompañó hasta la cátedra, invadiendo los claustros. El profesor inició su intervención con estas palabras: «Parece que esto huela a muerto, pero conste que si alguno muere, será la libertad de cátedra» ${ }^{48}$. Como había mucha gente que no cabía en el aula, decidieron continuar en el Paraninfo, hacia donde se dirigió la masa, si bien algunos mal intencionados, al pasar por las habitaciones del Rector, lanzaron muebles por las ventanas.

Los diputados por Barcelona Joan Sol i Ortega y Tiberio Ávila, ambos de Unión Republicana, al observar que las obras de Odón de Buen habían sido suprimidas de las que servían de texto en la Universidad, remitieron a Madrid el siguiente telegrama: «Ministerio Fomento.-Madrid. - Obras Odón de Buen Zoología y Geología aprobadas Consejo Instrucción Pública que desde

45 Nitrám, T. (1895), ¡Cosas de España!, La lectura dominical, año II, 91, 29 de septiembre, p. 6.

46 La lectura dominical, año II, 82, 6 de octubre de 1895, p. 10.

47 ARQUÉs (1984), p. 287.

48 BUEN (2003), p. 63. 
año 89 sirven de texto esta Universidad, acaban ser borradas cuadro asignaturas actual curso orden Rector instancia Obispo. Estimamos injusto y arbitraria esta resolución que además vulnera fueros ciencias libertad Cátedra independencia profesorado. Interesamos V. E. repare agravio volviendo fueros potestad civil» ${ }^{49}$.

Los tumultos prosiguieron los días siguientes, con agitaciones diarias y manifestaciones callejeras. El día 19 se reunió el consejo universitario, que formuló contra Odón de Buen los siguientes cargos:

Haber mantenido obras contrarias al dogma después del requerimiento del obispo.

Haber ocasionado el alboroto del día 7, saliendo a los claustros y pidiendo un local más amplio que el destinado á la clase.

Haber acudido a la Universidad el día 8, constándole que estaban suspendidas las clases, con lo cual desobedeció a la autoridad académica y dio margen á que fueran desacatados el rector y los catedráticos ${ }^{50}$.

¿Qué párrafos son los que habían incomodado a los miembros de la Iglesia Católica? Según dejó consignado el propio Odón de Buen, su Tratado elemental de Geología fue condenado por ciertos párrafos de la historia de las Ciencias Naturales con que comenzaba el tomo ${ }^{51}$. En mi opinión, los que reproduje anteriormente y que hacían referencia al largo período de dominación teocrática y a la permanente imposición de los conocimientos divinos. En la segunda edición, aparecida en 1896, se suprimió la parte histórica.

Sin embargo, en el Tratado, al tratar de la «evolución de los seres orgánicos» es posible encontrar otros fragmentos, como los que se reproducen a continuación, que también ocasionarían disgusto entre los miembros de la Iglesia Católica. Así, podemos leer que:

Simultánea a la evolución geológica trazada a grandes rasgos, se operaba otra en el seno de las aguas primero, después, también en la superficie de los continentes. La materia inorgánica, en el primitivo Océano, en función de aquellas excepcionales circunstancias, adquirió caracteres especiales, tendencias á la organización; se organizó más tarde, y la vida organizada fue recobrando formas variadisimas con las que hermoseó el planeta, contribuyendo a su evolución.

\footnotetext{
49 La Vanguardia, 4 de octubre de 1895, p. 2.

50 Contra D. Odón de Buen, El Imparcial, 20 de noviembre de 1895.

51 BUEN (2003), p. 65.
} 
En cada período geológico hubo una flora y una fauna, si no completamente nueva en sus detalles, con una fisonomía general característica ${ }^{52}$.

A continuación, Odón de Buen señala que estudiadas todas las formas animales y vegetales que se han sucedido en el tiempo, conviene anotar algunos hechos generales, como que primero aparecieron los animales y vegetales más rudimentarios; que los seres de mayor complicación son los últimos aparecidos en el tiempo; que existe una relación estrecha entre las circunstancias geológicas y climatológicas de cada período y los animales y vegetales que entonces vivieron; y cómo: «Desde que la vida apareció, se ha sucedido sin la más mínima interrupción, hasta hoy» ${ }^{53}$.

\section{LA REPOSICIÓN DE ODÓN DE BUEN Y LA EXTENSIÓN UNIVERSITARIA}

A Odón de Buen se le abrió el correspondiente expediente por el decano de la Facultad de Ciencias, José Ramón de Luanco, y fue llamado a Madrid por el Ministro de Instrucción Pública, Alberto Bosch y Fustegueras, si bien no pudo asistir por coincidir la llamada con el nacimiento de su hijo Fernando, y haberse complicado el puerperio de su esposa. Los alborotos no terminaron hasta el comienzo de las reglamentarias vacaciones de navidad. Al iniciarse el trimestre siguiente, Odón de Buen fue repuesto a su cátedra y él retomó su ingente labor docente.

Compuso entonces, una memorable Historia Natural, en edición popular pero profusamente ilustrada ${ }^{54}$, que comenzó a publicarse a comienzos de 1896, a razón de una peseta cada uno de los cincuenta cuadernos que la completaban ${ }^{55}$. En el prólogo de la obra, que dedica a la juventud iberoamericana, reafirma su compromiso con el uso responsable de la libertad de cátedra que las leyes españolas le concedían. $\mathrm{Al}$ respecto, en uno de los párrafos, decía:

Expongo la Ciencia tal como la entiendo; en el terreno ideal, ni vivo de prestado ni soy capaz de hipócritas habilidades para adaptarme el medio. Jamás impuse mi criterio á nadie; celoso de la libertad propia, nunca he pretendido menoscabar la Barcelona.

52 BUEN (1890), p. 294.

53 BUEN (1890), pp. 294-295.

54 Buen, O. de (c. 1896), Historia Natural, Edición popular (Con profusión de grabados), 55 Bibliografía, $A B C, 25$ de enero de 1896, p. 24. 
libertad ajena. Enemigo del magíster dixit, intento llegar a la conciencia de mis alumnos por la exposición fiel, nunca desfigurada, de los hechos demostrados. No cohibo materialmente á nadie, valiéndome de la autoridad oficial y de la amenaza de los exámenes, ni le engaño, falsificando los hechos, para ocultarles la verdad ${ }^{56}$.

Una prueba más de su compromiso con la educación a todos los niveles, fue su implicación en la Extensión Universitaria, que establecieron algunos catedráticos con los alumnos mejor preparados. Recorrieron Cataluña, durante muchos años, dando conferencias, y en Barcelona y en las poblaciones más populosas dieron cursillos con el más amplio y generoso criterio, cursillos que igual se desarrollaban en un círculo de recreo, que en un casino político u obrero de cualquier tendencia. También resultaron singulares las conferencias impartidas los sábados en círculos obreros, conferencias a las que concurrían los obreros con sus mujeres y su prole ${ }^{57}$. Esta actividad estaba en consonancia con otro mensaje que formuló en el prólogo de su Historia Natural: «Educaos, instruiros en las aulas, pero no seáis avaros de la Ciencia que poseáis, difundirla por el Pueblo; haced cuestión de honor arrancar á este de la ignorancia; lograréis así la grandeza de vuestra raza y contribuiréis al bienestar de la Humanidad» ${ }^{58}$.

Tras veintidós cursos académicos en la Universidad en Barcelona, por R. O. de 11 de octubre de 1911 consigue el traslado a Madrid. Los periódicos republicanos barceloneses le dedicaron afectuosas palabras de despedida. «Durante los muchos años en Barcelona se había distinguido por su propaganda a favor de la República y el libre pensamiento» ${ }^{59}$.

\section{CATEDRÁTICO EN MADRID ${ }^{60}$}

Dos razones debieron pesar en el traslado de Odón de Buen a Madrid, a la cátedra de Mineralogía y Botánica ${ }^{61}$. De un lado, el hecho de que llegaba a la

56 BUEN (c. 1896), p. xxi.

57 BUEN (2003), p. 60.

58 BuEN (c. 1896), t. 1, p. xviii.

$59 A B C, 10$ de noviembre de 1911, p. 14.

60 Debo agradecer la localización y copia del Expediente personal del catedrático de la Facultad de Ciencias Odón de Buen y del Cos, en el Archivo General de la Universidad Complutense (signatura: P. 453, 1), a mi doctoranda y colega Dolores Ruiz Berdún, competente e infatigable investigadora.

${ }_{61}$ La Subsecretaría de Universidades, en oficio de 11 de octubre de 1911, le dice al Rector de la Universidad Central: «En virtud de concurso de traslación; S M. el Rey (q. D. g.) ha 
máxima categoría en el escalafón, pues la de Madrid era la Universidad de término; de otro, su creencia de que desde Madrid (lo habría comprobado durante sus años de senador ${ }^{62}$, le sería más fácil impulsar la creación del Instituto de Oceanografía.

La cátedra, por la larga enfermedad y por el carácter de su antecesor, el reputado geólogo y mineralogista Salvador Calderón (1853-1911), llevaba unos cursos descuidada. En palabras del propio Odón de Buen «aquello no era una cátedra, era un meeting al que asistían muchos centenares de alumnos, cuantos cabían sentados y de pié en el amplio anfiteatro» ${ }^{63}$. Como el fallecimiento de Calderón también había dejado vacante el cargo de Director de la Escuela Botánica de la Universidad, el Rectorado acordó nombrar a Odón de Buen Director de dicha Escuela ${ }^{64}$.

En poco tiempo, el nuevo catedrático logró la atención de los alumnos y las cosas cambiaron. Puso en acción en la cátedra madrileña un plan similar al que había llevado a cabo en Barcelona, y que ya hemos referido. Para las lecciones orales, a las que acudían alumnos de las licenciaturas de Medicina, Farmacia, Ciencias y Arquitectura, el elevado número resultaba un inconveniente. También lo era para las prácticas, ya que al llegar no encontró ni un local suficientemente amplio, ni la cantidad suficiente de material, un material que además era más propio para la investigación que para la enseñanza. «El salón en que estaban las colecciones se convirtió pronto en una sala de manipulaciones con instalación apropiada de gas y electricidad, con numerosos sopletes automáticos y las prácticas de Mineralogía se daban bastante bien, aunque eran pocas e insuficientes, porque a la vez podían trabajar a lo sumo cincuenta alumnos» ${ }^{65}$.

Como también había hecho en Barcelona, en Madrid, regularmente, publicaba el programa de la asignatura. Así, al revisar el Programa del curso de Geología, Mineralogía y Botánica del año académico 1912-191366, vemos

resuelto nombrar a D. Odón de Buen y del Cos Catedrático numerario de Mineralogía y Botánica en la Facultad de Ciencias de esa Universidad con el haber anual de ocho mil pesetas y demás ventajas de la Ley...».

62 Odón de Buen había sido senador en la legislatura 1907-1908 por la provincia de Barcelona.

63 BUEN (2003), p. 124.

64 El nombramiento se produjo con fecha 10 de noviembre de 1911, el día antes de la toma de posesión de la cátedra. Expediente personal del catedrático.

65 BUEN (2003), pp. 56-57.

66 Buen, O. de (1912a), Programa del curso de Geología, Mineralogía y Botánica (con claves para determinar minerales y familias botánicas). Curso de 1912 á 1913, Madrid, Librería General de Victoriano Suárez. 
que este constaba de ciento diez lecciones orales, divididas en dos bloques, las cincuenta y cinco primeras para el de geología y las cincuenta siguientes para el de botánica. El cuestionario de ejercicios prácticos se reducía a diez de geología y diez de botánica. Las prácticas de geología incidían en la determinación de cristales y en diferentes ensayos con minerales, además de la determinación de fósiles. Por su parte, las botánicas llevaban a cabo la preparación y determinación de preparaciones microscópicas, el estudio morfológico de diferentes partes de la flor y la determinación de familias botánicas con clave dicotómica. Generalmente, las prácticas se verificaban por las tardes, divididos los alumnos en diferentes grupos y figurando como responsable, de cada uno de ellos, un alumno sobresaliente ${ }^{67}$. Por su parte, en la minuta de excursiones se recogían cuatro: a las minas de Almadén, a Mónaco, a Córdoba, Málaga, etc. y a Cuenca y la Ciudad Encantada, que comentaremos dentro de un momento.

Continuó en Madrid la edición de textos universitarios. En 1912 publica el Nuevo resumen de Geología general y de España ${ }^{68}$, que constaba de una introducción y seis partes (Morfología terrestre, Geognosia, Dinámica terrestre, Geología Histórica, Geología de España y Prehistoria). Al año siguiente, el Nuevo resumen de Botánica general, con los fundamentos de la biología y la parasitología vegetal ${ }^{69}$.

\section{LAS EXCURSIONES DESDE MADRID}

Como había sido su norma hasta entonces, en Madrid de Buen siguió programando dos tipos de excursiones, las que ocasionaban gastos, que eran voluntarias, y las gratuitas, que eran obligatorias y se limitaban a los alrededores de Madrid, en grupos de 25 a 30, con el profesor, los auxiliares y los ayudantes de grupo. Advertía en el programa que: «En las excursiones forman los alumnos el herbario escolar. Los que no lo presenten en el examen ó no conste que lo han formado, tendrán que determinar siquiera las familias de algunas plantas $\rangle^{70}$.

67 Buen (1912a), pp. 8-9.

68 BuEn, O. de (1912b), Nuevo resumen de Geología general y de España, Madrid, Librería Gutenberg de José Ruiz.

69 Buen, O. de (1913a), Nuevo resumen de Botánica general, con los fundamentos de la biología y la parasitología vegetal, Madrid, Librería General de Victoriano Suárez.

70 Buen (1913a), p. 10. 
Ya en el primer curso en Madrid, el de 1911-1912, organizó excursiones a la Ciudad Encantada de Cuenca, a las minas de Almadén y a Barcelona, Mallorca, Alicante y Elche. Excursiones para las que aprovechaba los días festivos. Así en el Programa del curso 1912 a 1913 puede leerse:

A las Minas de Almadén

En el mes de Noviembre, aprovechando los días 1, 2 y 3 que son fiesta. Si como en el curso último, el número de alumnos impone la división en dos grupos, el grupo primero saldrá de Madrid la tarde del jueves 31 de octubre, permaneciendo en Almadén hasta el sábado y el grupo segundo saldrá el viernes $1^{\circ}$ de Noviembre, para regresar el lunes 4 , por la mañana... ${ }^{71}$

Entre los objetivos de esta excursión a Almadén, donde siempre eran bien acogidos y acompañados por los ingenieros, estaban el estudio de las minas, el proceso de obtención del mercurio, el estudio de los territorios silúrico y devónico, la recolección de fósiles y la realización de herborizaciones. Odón de Buen recordaba en sus memorias cómo: «Se complacían los estudiantes en meter las manos en los depósitos del metal líquido y a pesar de mis advertencias (y quizá para comprobarlas) muchos perdieron sus sortijas, es sabido que el mercurio amalgama el oro» ${ }^{72}$.

En aquel Programa del curso 1912 a 1913, la excursión por Andalucía se proyectaba para la Semana Santa y Pascua, con el objeto de apreciar su riqueza geológica y su espléndida flora, como espléndidos son sus monumentos y paisajes. Si las circunstancias lo permitían, estaba previsto que los excursionistas visitaran Melilla y su campo ${ }^{73}$.

Pero, desde Madrid, la excursión a la Ciudad Encantada de Cuenca era la que resultaba verdaderamente atractiva para Odón de Buen. De modo que no dejaba transcurrir curso académico sin llevar a sus alumnos a este paraje natural de formaciones calcáreas formado a lo largo de miles de años, donde el agua es el verdadero artífice del modelado, pero donde también las rocas presentan especiales condiciones para el modelo. De ahí que en un artículo sobre la Ciudad Encantada de Cuenca, publicado por Odón de Buen en la revista Blanco y Negro en 1913, consignara cómo: «En pocos lugares de la tierra la acción secular del agua, lluvia o nieve, arroyo ó torrente, ha producido más espléndido modelado que en la ciudad encantada de Cuenca» ${ }^{74}$.

71 BUEN (1913a), p. 28.

72 BUEN (2003), p. 126.

73 BUEN (1912a), p. 29.

74 Buen, O. de (1913b), La Ciudad Encantada de Cuenca, Blanco y Negro, 15 de junio, pp. 16-17, p. 17. 
Dicho artículo que repasa las condiciones geológicas que han hecho posibles los cambios en «nuestro viejo planeta» y en donde señala cómo las «potentes masas calizas de aquel terreno se depositaron mansamente en un largo período de calma, durante la edad secundaria», termina con la recomendación del docente: «No puede ofrecerse cátedra mejor á los estudiantes para el conocimiento de la Naturaleza. Por eso realizo la excursión anual á la Ciudad encantada $\rangle^{75}$.

El 11 de junio de 1929 la Ciudad Encantada sería declarada Sitio Natural de Interés Nacional y en 1934, en plena República, se celebró alló un homenaje a Odón de Buen, a Luis Martínez Kleiser y a Juan Jiménez Aguilar, con el objeto de que, a partir de ese momento, tres calles del paraje llevaran sus nombres ${ }^{76}$.

Algunos otros puntos singulares próximos a Madrid, como Sigüenza, Toledo y El Escorial, también eran objeto de excursión algunos años. En Sigüenza los alumnos recogían aragonitos y otros minerales, así como fósiles; en Toledo buscaban conchas marinas sobre la playa que había descubierto, no mucho tiempo antes, el naturalista y matemático Ventura Reyes Prósper (1863-1922), que en algunas ocasiones les acompañaba; en El Escorial, el objetivo era la localización de buenos ejemplares de cristal de roca y feldespatos, al tiempo que se prestaba atención a la flora y a los efectos sorprendentes de las erosiones del granito por la acción de los agentes atmosféricos ${ }^{77}$.

Las excursiones que se proyectaban a zonas más alejadas con frecuencia se anunciaban en la prensa de la época. Así en el diario $A B C$ del jueves 17 de diciembre de 1914 encontramos el siguiente aviso:

La excursión escolar científica organizada por el catedrático de la Universidad Central D. Odón de Buen se realizará del 26 de Diciembre al 4 de Enero, siguiendo el siguiente itinerario:

Madrid, Alicante, Palma de Mallorca, Miramar, Soler, Grutas de Manacor, Laboratorio biológico de Porto-Pi, Ibiza, Alicante, Elche. Madrid.

$\mathrm{Si}$ algún estudiante desea inscribirse, todavía puede hacerlo, concurriendo el sábado próximo, día 19, al Laboratorio de Mineralogía y Botánica de la Universidad, a las cuatro y media de la tarde ${ }^{78}$.

75 BUEN (1913b), p. 17.

76 ABC de Sevilla, 31 de agosto de 1934, p. 39.

77 BUEN (2003), pp. 126-127.

$78 A B C, 17$ de diciembre de 1914, p. 21. 
En ocasiones, la prensa recoge la salida o llegada de los excursionistas a algún lugar. Así, el 14 de diciembre de 1913 el $A B C$ anuncia la salida para Mónaco de la excursión escolar de la cátedra de Mineralogía y Botánica, organizada y dirigida por el «sabio catedrático» ${ }^{79}$. Quince días después, el mismo diario insertaba una foto a media página de los expedicionarios en el del Museo de Mónaco, con el siguiente pié: «Estudiantes españoles en el extranjero. El sabio catedrático D. Odón de Buen y un grupo de sus alumnos de la Universidad de Madrid en la terraza del Museo Oceanográfico de Mónaco» ${ }^{80}$. Un último ejemplo, el 3 de mayo de 1919, el $A B C$ insertaba una nota fechada en San Sebastián el día de antes, donde se señalaba que había llegado la expedición científica compuesta de 37 alumnos y dirigida por Odón de Buen, que habían sido recibidos por miembros de la Sociedad Oceanográfica y que al día siguiente continuarían viaje a París ${ }^{81}$.

\section{LA CÁTEDRA DE BIOLOGÍA Y LOS ÚLTIMOS AÑOS DE CATEDRÁTICO EN MADRID}

Pocos años después, a comienzos del curso 1923-1924, Odón de Buen pasó a la cátedra de Biología ${ }^{82}$, si bien durante algunos cursos estuvo encargado también de la de Geología ${ }^{83}$. Para la organización de las enseñanzas de la nueva disciplina tuvo en cuenta cómo se daba su enseñanza en algunas universidades extranjeras que él había visitado, como en los preparatorios de Jena y París, sin embargo el resultado obtenido no le satisfizo completamente, de ahí que en sus Memorias consignara:

en casi medio siglo de catedrático no pude, dicho con mayor exactitud, no me dejaron organizar las enseñanzas como yo era capaz de organizarlas, a la altura de las mejores. La atención principal y los medios en mayor escala deben emplearse en las clases prácticas, pero estas son penosas para el profesor y muy caras para la Universidad o para el Estado. Se necesita cuantioso instrumental, amplios locales llenos de luz del día y bien iluminados en las tardes del invierno; mobiliario cómo-

\footnotetext{
$79 A B C, 14$ de diciembre de 1913, p. 18.

$80 A B C, 29$ de diciembre de 1913, p. 4.

$81 A B C, 3$ de mayo de 1919, p. 25.

82 R. O. de 22 de septiembre de 1923, Boletín Oficial del Ministerio de Instrucción Pública y Bellas Artes, n. ${ }^{\circ} 80$ (5 de octubre de 1923).

83 R. O. de 17 de octubre de 1923, Boletín Oficial del Ministerio de Instrucción Pública y Bellas Artes, n. ${ }^{\circ} 88$ (2 de noviembre de 1923).
} 
do para los alumnos y una verdadera legión de ayudantes o preparadores bien retribuidos $^{84}$.

Todavía antes de jubilarse, y gracias a la relativa autonomía económica que se concedió a las facultades y al mayor presupuesto de que dispusieron, pudo obtener un crédito extraordinario y construir una amplio laboratorio con mucha luz, cómodas mesas de trabajo (esmaltadas en blanco), con capacidad para cerca de un centenar de estudiantes. Para ello utilizó, transformándola, la vetusta cátedra de Zoología, dando las clases orales en el gran aula del pabellón del jardín. Un día visitó la Universidad el Ministro de Instrucción Pública que, impresionado del contraste de este moderno laboratorio con el resto del edificio, preguntó de qué se trataba. Le dijeron que era el laboratorio de Biología que Odón de Buen había instalado, lo que satisfizo a nuestro protagonista, aunque para dentro pensara: «¿por qué no me han dejado hacer esto mismo hace veinte años?» ${ }^{85}$.

A propuesta de la Facultad de Ciencias de la Universidad Central, la Subsecretaría de Universidades, en diciembre de 1931, le encargó, por acumulación, la Cátedra de Complementos de Biología para Medicina ${ }^{86}$. Y, mientras tanto, seguía desempeñado numerosas comisiones en el extranjero. Una de las últimas, en sus años de catedrático, le incorporó a la delegación española en la $3^{a}$ Conferencia Hidrógráfica Internacional que se reunió en Mónaco en el mes de abril de 1932. Como era perceptivo, Odón de Buen se dirigió al Decano de la Facultad de Ciencias señalándole que formaba parte de la misma por disposición ministerial y que, para que los servicios de la cátedra no quedaran interrumpidos, Jesús Maynar se encargaría de las clases orales de Complementos de Biología, Fernando de Buen y Lozano de las de Biología para alumnos de Ciencia y que Cayetano escribano seguiría al frente de las prácticas ${ }^{87}$.

El 18 de noviembre de 1933 cumplió los setenta años de edad, la edad reglamentaría para la jubilación. Como el curso había comenzado pocas semanas antes, los alumnos solicitaron su continuación en la cátedra hasta la finalización del curso, lo que consiguió tras el favorable informe del Claustro de la Facultad de Ciencias (Gaceta de Madrid, 23-XI-1933). Meses después, concretamente el 4 de febrero de 1934, amigos, colegas y antiguos discípulos

84 BUEN (2003), p. 129.

85 BUEN (2003), p. 122.

86 Expediente personal del catedrático. Odón de Buen y del Cos. Archivo General de la Universidad Complutense, Signatura P. 453, 1.

87 Archivo de la Universidad Complutense, signatura 161/07-012. 
le dispensaron un emocionante banquete. Con el agasajado tomaron asiento en la presidencia el Ministro de Trabajo, José Estadella; el Subsecretario de Instrucción Pública, señor Armasa; el ex Ministro, José Giral; el Rector de la Universidad Central, doctor Cardenal; el Director del Instituto Geográfico y Catastral, señor Gastardi; el Decano de la Facultad de Medicina, señor Sánchez Cobisa; el Director de la Casa de Velázquez, Mr. Dumas, un ayudante del Ministro de Marina y los doctores Folch, Sloker, Vich y Martínez Álvarez ${ }^{88}$.

Ofreció el acto, con palabras llenas de afecto y cordialidad para el agasajado el doctor Rafael Folch, de la Facultad de Farmacia. Después hablaron el subsecretario de Instrucción Pública y el ministro de Trabajo, quienes también exaltaron la personalidad científica del doctor Odón de Buen ${ }^{89}$. El agasajado, en su discurso, hizo un repaso a su larga carrera como investigador y como docente, señalando en un momento determinado que:

Desde los primeros años de mi vida académica inspiré mis enseñanzas en el método experimental; este método es hoy axiomático, pero entonces ique difícil y aun peligroso era seguirle! Porque los profesores de la vieja cepa nos odiaban a los nuevos cordialmente; de mis libros, muchas familias destruían las hojas que trataban de las funciones de reproducción y de la embriogenia; los castraban, sin duda, para que fueran eunucos; para juzgar la poda tened en cuenta que la mayoría de mis alumnos eran de Medicina ${ }^{90}$.

\section{LA ÚLTIMA CLASE EN LA UNIVERSIDAD DE BARCELONA}

Luego de que el propio Odón de Buen manifestara su deseo de impartir la última clase en la Universidad de Barcelona, concretamente en el mismo aula donde cuarenta y cinco años antes iniciara su andadura como catedrático universitario, al mediodía del sábado 26 de mayo, de ese mismo año de 1934, pudo Odón de Buen cumplir su deseo y dictar su última lección en el ámbito de la Universidad ${ }^{91}$. Bajo la presidencia del Rector, Pere Bosch Gimpera (1891-1974), y con un aula que se encontraba llena de estudiantes y ex alumnos suyos, así como un buen número de catedráticos, programa en mano, Odón de Buen repitió lo más fielmente posible aquella primera lección, al

88 La Vanguardia, 4 de febrero de 1934.

89 La Vanguardia, 4 de febrero de 1934.

90 BuEN (2003), p. 157.

91 En la Facultad de Ciencias la última lección del Doctor Odón de Buen, La Vanguardia, 27 de mayo de 1934, p. 9. 
tiempo que recordó a los compañeros que habían desaparecido y agradeció, a todos los reunidos, la asistencia al acto de su despedida de la Universidad ${ }^{92}$.

El decano de la Facultad de Ciencias, Eduardo Fontseré, pronunció unas breves palabras, en contestación a las pronunciadas por Odón de Buen, en donde señaló que la Facultad había tomado el acuerdo de nombrarle catedrático honorario de la misma. De Buen aceptó con emoción el nombramiento y se comprometió, si las fuerzas no le faltaban, a dar todos los años un curso de Oceanografía, disciplina que aún no estaba presente en la Universidad. Cerró el acto el Rector de la Universidad, Bosch Gimpera, que le dedicó cariñosas palabras. A primera hora de la tarde, Odón de Buen fue obsequiado con una comida en el Hotel Colón, ofrecida por los catedráticos y alumnos de la Facultad de Ciencias $^{93}$.

\section{EPÍLOGO}

Tras la jubilación de Odón de Buen, lo que deberían haber sido unos años reposados de jubilo en Zuera, en «una casa de arquitectura y comodidades modernas $\rangle^{94}$, donde hubiera podido redactar con calma sus memorias, por el transcurrir de la guerra se convirtieron en unos años traumáticos, en los que sufrió detención en Palma de Mallorca, hubo de enterarse del fusilamiento de su hijo Sadi así como exiliarse en México D.F., donde fallecería el 3 de mayo de 1945, con 81 años de edad. En España, su labor como catedrático comprometido con la biología evolucionista sería silenciada.

Recibido: 22 de septiembre de 2010.

Aceptado: 15 de febrero de 2011.

\footnotetext{
92 BUEN (2003), pp. 158-159.

93 BUEN (2003), p. 159 y En la Facultad de Ciencias...

94 BUEN (2003), p. 17.
} 